\title{
O processo de implantação de diretrizes na prática médica
}

\author{
Fabio B. JATENE* Wanderley Marques BERNARDO*, \\ Rosangela MONTEIRO-BONFÁ*
}

RBCCV 44205-534

\begin{abstract}
Jatene F B, Bernardo W M, Monteiro-Bonfá R - O processo de implantação de diretrizes na prática médica. Rev Bras Cir Cardiovasc 2001; 16(2): 89-93.

RESUMO: Diretrizes tradicionalmente são desenvolvidas por médicos para aumentar a qualidade dos cuidados ao paciente, podendo fornecer aos seus usuários informação médica valiosa, aumentando a objetividade na decisão médica. Este artigo aborda aspectos determinantes de diretrizes de boa qualidade, sobretudo os métodos de elaboração, discutindo fatores envolvidos em sua aplicabilidade como custos implicações éticas e legais.
\end{abstract}

DESCRITORES: Diretrizes. Recomendações médicas. Ética médica.

\section{INTRODUÇÃO}

No Brasil, a Medicina vem sofrendo um processo de profundas modificações, na tentativa de solucionar problemas decorrentes, por exemplo, do aumento do número de escolas médicas, que lançam, anualmente, centenas de profissionais, sobretudo nos grandes centros, gerando dificuldades na padronização e na qualidade do atendimento. Além disso, de forma semelhante, enfrentam-se limitações no preparo adequado de especialistas, bem como, crescente pressão por parte das fontes pagadoras, buscando redução de custos.

Paralelamente, a Medicina mundial encontrase em um momento de globalização, onde grande número de publicações é produzido e difun- dido diariamente, ao mesmo tempo permitindo acesso instantâneo, mas em contrapartida, inviabilizando a assimilação de conhecimentos na mesma velocidade. Da mesma forma, os pacientes chegam, aos nossos consultórios, melhor informados sobre os tratamentos disponíveis e a tecnologia existente, resultado da ampla difusão destes conhecimentos.

Muitas destas informações disponíveis são conflitantes entre si, promovendo condutas distintas para a mesma situação, necessitando uma análise crítica sobretudo quanto ao rigor científico e aos possíveis interesses particulares envolvidos.

Dentro deste contexto, surge um processo de busca de otimização do atendimento ao paciente, no qual a literatura médica tem exercido impor-

\footnotetext{
Trabalho realizado no Instituto do Coração do Hospital das Clínicas da Faculdade de Medicina da Universidade de São Paulo. São Paulo, SP, Brasil. Recebido para publicação em janeiro de 2001

* Do Instituto do Coração do Hospital das Clínicas da Faculdade de Medicina da Universidade de São Paulo.

Endereço para Correspondência: Fabio B. Jatene. Instituto do Coração. Divisão de Cirurgia. Av. Dr. Enéas de Carvalho Aguiar, 44. Cerqueira César. São Paulo, SP, Brasil. CEP: 05403-001. Tel/Fax: (11) 3069-5248. e.mail: fabiojatene@incor.usp.br
} 
Jatene F B, Bernardo W M, Monteiro-Bonfá R - O processo de implantação de diretrizes na prática médica. Rev Bras Cir Cardiovasc 2001; 16(2): 89-93.

tante papel através da geração de normas de conduta médica (diretrizes). A pedra angular de sustentação desse processo em bases científicas foi estabelecida pelo Evidence-Based Medicine Working Group. Este grupo fez a primeira publicação, em 1992, usando a denominação de "Medicina Baseada em Evidência", iniciando a série do JAMA "User's Guide to the Medical Literature" (1).

Métodos formais para o desenvolvimento de diretrizes têm surgido para atender às necessidades das Sociedades Médicas, com o intuito de minimizar a variação da pratica médica, diminuir os custos, monitorar os cuidados inapropriados, auxiliar os médicos atualizados com as novas informações, estabelecer pesquisas prioritárias e promover melhores resultados no atendimento ao paciente. Métodos baseados em evidências garantem que diretrizes proporcionam recomendações válidas baseadas em uma avaliação crítica da melhor evidência disponível, em detrimento ao processo baseado em opinião informal (2).

Atualmente, numa iniciativa da Associação Médica Brasileira e do Conselho Federal de Medicina, foi desencadeado um processo junto "as Sociedades Médicas Brasileiras, denominado "Projeto Diretrizes". Este projeto prevê a criação de diretrizes de prevenção, diagnóstico e tratamento de doenças mais prevalecentes em nosso meio, baseadas nas evidências científicas atualmente disponíveis na literatura nacional e internacional, que determinarão os graus de recomendação.

Cerca de 50 sociedades de especialidades médicas estão envolvidas no projeto, sendo orientadas a realizar uma busca sistemática na literatura,

\section{TABELA 1}

GRAU DE RECOMENDAÇÃO E NÍVEL DE EVIDÊNCIA ESTUDOS TERAPÊUTICOS E PREVENTIVOS

A 1 Revisão sistemática de ensaio controlado e aleatorizado \& megatrial

2 Ensaio controlado e aleatorizado de intervalo de confiança pequeno

3 Outros ensaios clínicos

4 Revisão sistemática de estudos de coorte

B 5 Estudo de coorte

6 Coorte de cuidados médicos recebidos (outcomes research)

7 Revisão sistemática de estudos caso controle

8 Estudo caso-controle

C 9 Série de casos

D 10 Consenso ou opinião de especialistas da melhor evidência científica disponível para cada conduta apresentada.

A Sociedade Brasileira de Cirurgia Cardiovascular está participando deste processo com a elaboração de 3 diretrizes:

1. Tratamento cirúrgico da insuficiência coronária.

Coordenadores: Jarbas J. Dinkhuysen e Ricardo Carvalho Lima

2. Tratamento cirúrgico das doenças da aorta.

Coordenadores: Domingo M. Braile e José Honório de A. P. Fonseca

\section{Tratamento cirúrgico das doenças valvares.}

Coordenadores: Francisco Gregori Junior e Pablo Maria A. Pomerantzeff.

As recomendações são feitas em graus $A, B, C$ e $D$, segundo a força de evidência científica das referências bibliográficas que vão de 1 a 10 . A recomendação científica mais forte é a $A$ e a com menor peso, baseada apenas em consenso ou opinião de especialista, é a D (Tabelas 1 e 2).

Os fatores determinantes de diretrizes de boa qualidade, conferindo-Ihes legitimidade e confiabilidade, estão relacionados com a forma de elaboração e aplicabilidade destas.

Dentro do item elaboração serão analisados aspectos como a autoria e o método utilizado; além disso, os custos, as implicações éticas e legais determinantes da aplicabilidade das diretrizes serão abordados a seguir.

\section{TABELA 2}

GRAU DE REDOMENDAÇÃO E NÍVEL DE EVIDÊNCIA ESTUDOS DIAGNÓSTICOS

\footnotetext{
A 1 Revisão sistemática de estudos melhor desenhados

2 Padrão ouro + observação cega + seleção adequada

3 Alta sensibilidade e/ou especificidade

4 Revisão sistemática de estudos pior desenhados

B 5 Padrão ouro + observação cega ou seleção 6 adequada

7

8 Observação cega + seleção adequada sem padrão ouro

C 9 Padrão ouro ou observação cega ou seleção adequada

D 10 Consenso ou opinião de especialistas
} 
Jatene F B, Bernardo W M, Monteiro-Bonfá R - O processo de implantação de diretrizes na prática médica. Rev Bras Cir Cardiovasc 2001; 16(2): 89-93.

\section{DEFINIÇÃO E MÉTODOS DE ELABORAÇÃO}

Diretrizes são um conjunto de recomendações estruturadas, periodicamente atualizadas, cujo principal objetivo é produzir ações de maior qualidade.

Mundialmente recebem a denominação de guidelines, sendo também, erroneamente, usadas como sinônimo de consenso.

Diretriz, protocolo, guideline ou guia de conduta basicamente significam a mesma coisa, com pequenas nuances. No protocolo, guideline ou guia de conduta o que está escrito deve ser seguido. Diretriz é algo que se gostaria que fosse seguido, mas há uma maior preocupação com a autonomia do médico.

O consenso é apenas um dos métodos de elaboração de diretrizes, podendo ser formal ou informal. O consenso, apesar de ser o mais utilizado, é o método menos adequado, pois caracteriza-se por ausência de metodologia, dependendo da dinâmica de um grupo e de convicções pessoais.

A Associação Médica Brasileira recomenda que as diretrizes elaboradas devem apresentar as seguintes características:

- Orientação diagnóstica, terapêutica e preventiva;

- Não definir doença etiopatogenia e fisiopatologia;

- Abordar os temas de maior importância e freqüência;

- Aplicáveis em todo o território nacional;

- Contemplar, mas não obrigar o uso de recursos e tecnologias avançadas;

- Não devem gerar conflito ético entre médicos e pacientes;

- Contemplar eventuais opções, permitindo o bom senso;

- Devem ser baseadas em evidências científicas;

- A determinação do nível de evidência depende do desenho da pesquisa;

- Estabelece graus de recomendação (A,B, C e D)

- Recomendado basear-se em revisões sistemáticas e ensaios clínicos aleatorizados;

- Estudos observacionais são aceitáveis;

- Séries de caso podem ser excepcionalmente considerados;

- Opinião ou consenso de especialistas devem ser evitados como fonte exclusiva.

O método ideal de elaboração é aquele que inclui os seguintes itens:
- Baseado em evidência científica;

- Considera benefícios, riscos, custos e realidade local;

- Estima probabilidade do resultado

$\mathrm{Na}$ busca de evidências científicas, deve-se analisar criticamente os dados disponíveis na literatura, avaliando a metodologia empregada e interpretando os resultados.

A autoria das diretrizes merece particular atenção, já que deverá estar isenta de interesses de outros grupos, pois a prioridade é o paciente e meIhoria ao seu atendimento. Elaborando diretrizes, agentes pagadores, sejam estes governamentais ou privados visam, muitas vezes, redução de custos, invadindo, muitas vezes, a autonomia médica. Por outro lado, a indústria procura conquistar e ampliar mercados e os grupos profissionais, estender a sua influência.

A instituição de um comitê elaborador, com a participação das Sociedades Médicas, minimiza a influência de interesses de grupos, além disso, utilizando rigor metodológico é a forma ideal de gerar diretrizes. O grupo elaborador deve ter em mente as amplas implicações nos Serviços de saúde, garantindo a aplicabilidade das diretrizes.

\section{APLICABILIDADE}

\section{Custos}

Dentro dos principais fatores limitantes da aplicabilidade das diretrizes, encontram-se aqueles relacionados aos custos de sua implantação. Muitas vezes, requerem aquisição de novos conhecimentos e habilidades, bem como treinamento de profissionais.

A necessidade de adaptações institucionais, envolvendo recursos humanos e financeiros, determinando a obtenção de novas tecnologias, pode inviabilizar a disseminação de uma diretriz na prática médica diária.

Algumas questões devem ser consideradas, por exemplo, como:

- qual a aplicabilidade de diretrizes cujo desenho baseou-se em megatrials?

- os resultados obtidos em trials são reproduzíveis na prática clínica?

- na rápida adoção de novos tratamentos, determinados pelas diretrizes, há avaliação prévia de custos e da real eficiência desta terapêutica na população em geral? 
Jatene F B, Bernardo W M, Monteiro-Bonfá R - O processo de implantação de diretrizes na prática médica. Rev Bras Cir Cardiovasc 2001; 16(2): 89-93.

Devemos considerar que, na prática clínica diária, os recursos são mais limitados, não reproduzindo, muitas vezes, os resultados obtidos em estudos controlados aleatorizados.

Além disso, tratamentos caros, podem ganhar aplicação imediata, quando, na prática, seus resultados podem ser inferiores aos esperados.

Dentro do aspecto custo, a adoção de um novo tratamento depende de uma relação de equilíbrio entre a melhoria dos resultados obtida e o incremento de custos determinado.

HAYCOX et al. (3), em 1999, através de um esquema ilustrativo de áreas representando custos maiores ou menores e resultados melhores ou piores, com suas interfaces, procuraram modelar a adoção de diretrizes (Figura 1).

Assim, as recomendações podem ser positivas ou negativas, levando em consideração, prioritariamente, a obtenção dos melhores resultados, e sempre que possível, a redução de custos.

\section{ASPECTOS ÉTICOS E LEGAIS}

O processo de implementação de diretrizes abrange aspectos éticos, onde a relação médico
- paciente deve ser preservada. Deve-se evitar a produção de reações negativas entre os pacientes, impedindo conflitos de interesses entre médico e paciente.

As recomendações devem considerar os possíveis riscos aos pacientes, respeitando suas características individuais, e do mesmo modo, normas e valores regionais.

Igualmente, as diretrizes devem garantir a autonomia médica e a liberdade de decisão, constituindo-se em recomendações, baseadas em evidências científicas, para a maioria dos pacientes e não, obrigatoriamente, regras para todos (4).

A essência das diretrizes é ética. Entretanto, a aplicação de valores não-médicos associada à intensa pressão das fontes pagadoras pode criar um ambiente favorável ao uso inadequado e/ou manipulação destas ${ }^{(5)}$.

Outro ponto importante diz respeito aos aspectos legais das diretrizes.

Nos Estados Unidos e na Europa, as diretrizes começam a ser utilizadas como substrato em decisões judiciais. Diretrizes podem ser apresenta-

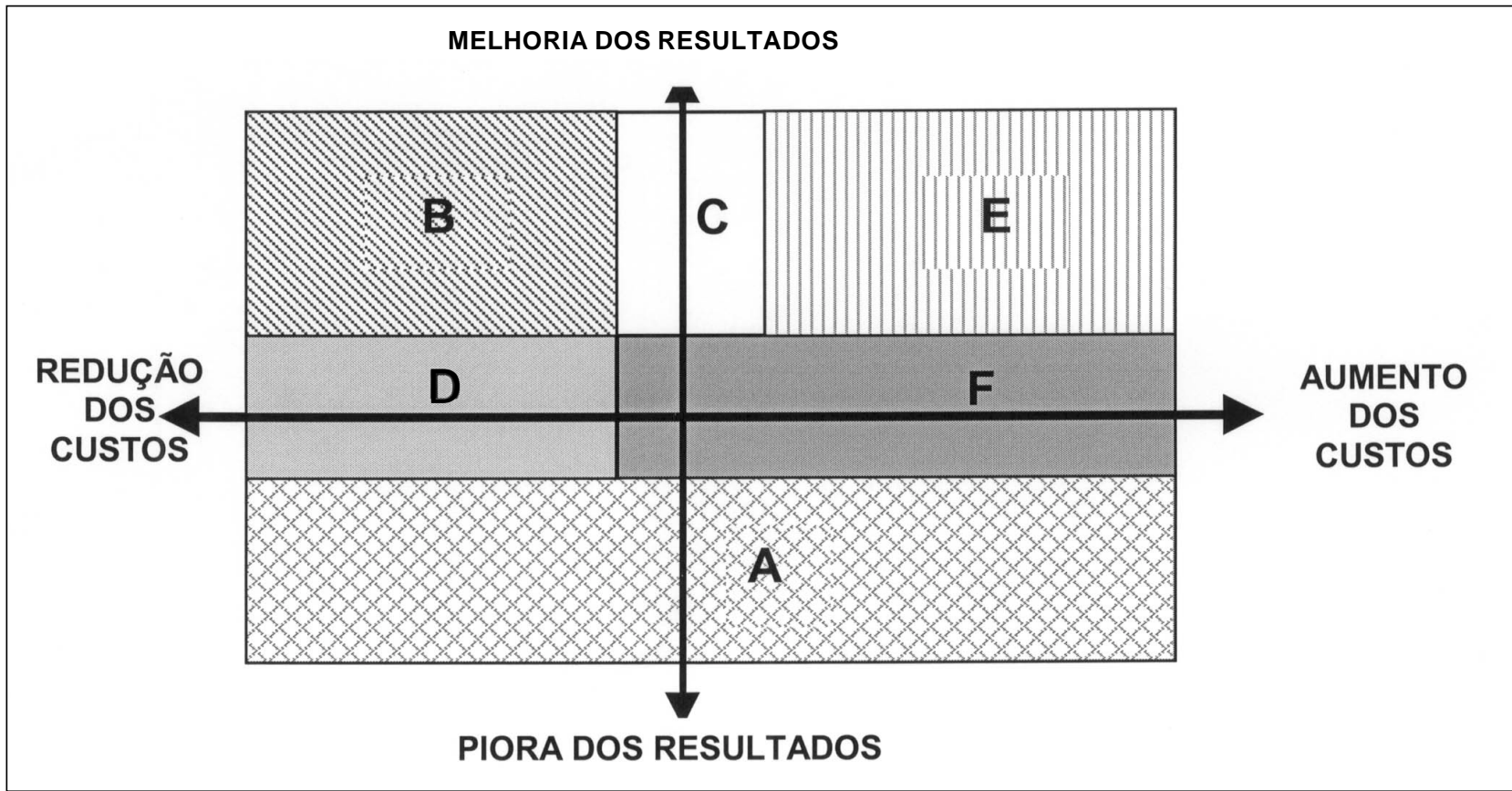

Fig. De acordo com as áreas representadas neste diagrama podemos depreender que, na área:

A - a recomendação é sempre negativa;

B - - a recomendação é sempre positiva;

C - a recomendação é posivita, mas pode envolver custos extras;

D e F - a recomendação é negativa, podendo, excepcionalmente, se constituir em opção aidiconal custos extras;

$\mathrm{E} \quad-$ a recomendação é positiva, mas com maiores custos, gerando resistência junto às fontes pagadoras (modificando de HAYCOX et al. ${ }^{3}$ ) 
Jatene F B, Bernardo W M, Monteiro-Bonfá R - O processo de implantação de diretrizes na prática médica. Rev Bras Cir Cardiovasc 2001; 16(2): 89-93.

das à Justiça como evidência de cuidados padronizados, aceitáveis e habituais, entretanto, não podem substituir o testemunho de um especialista. A Justiça não adota as recomendações preconizadas em diretrizes como "padrão-ouro" legal porque a simples existência das mesmas não estabelece que a concordância com elas é sinônimo de absolvição e que a discordância sinônimo de negligência.

Em casos de resultados desfavoráveis, nos quais as diretrizes foram seguidas, de quem é a responsabilidade legal: do praticante ou do autor? Nos Estados Unidos, conflitos entre diretrizes e opinião médica têm levado a Justiça estabelecer que os profissionais ou praticantes não podem se defender de acusação de negligência, argumentando que o seu julgamento clínico foi influenciado negativamente pelas diretrizes.

HYAMS et al. (6) analisaram 259 processos médicos existentes nos Estados Unidos, em um período de 2 anos, e constataram que as diretrizes foram úteis na absolvição em 4 casos e decisivas para condenação em outros 12 processos.

\section{CONSIDERAÇÕES FINAIS}

Em resumo, as diretrizes devem proporcionar uma melhoria real no atendimento ao paciente, auxiliando nas decisões médicas, contemplando a autonomia e permitindo a avaliação crítica por quem as utiliza.

Este é um momento muito importante, de profundas mudanças na prática médica em nosso país, do qual não podemos ficar à margem. Como já dito anteriormente, nossa Sociedade está envolvida neste processo, preparando 3 diretrizes que deverão estar prontas em alguns meses.

As diretrizes, muitas vezes, geram resistência por parte de alguns colegas porque prevêem mudanças de comportamento, que teoricamente poderiam cercear a autonomia médica. Entretanto, devem ser encaradas como um instrumento de auxílio nas decisões clínicas, podendo sofrer alterações advindas da experiência adquirida com sua aplicação na prática médica diária. Assim, devem ser criados mecanismos para produzir diálogo entre os responsáveis pela elaboração e todos os membros de nossa Sociedade. Nossa Revista pode ser um importante instrumento de comunicação, recebendo sugestões e balanços iniciais após a instituição e aplicação dessas primeiras diretrizes.

Jatene F B, Bernardo W M, Monteiro-Bonfá R - Implementation process of clinical practice recommendations on surgery. Rev Bras Cir Cardiovasc 2001; 16(2): 89-93.

ABSTRACT: Traditionally, guidelines are developed by physicians to improve quality of health care, to provide practitioners with valuable medical information and to improve objectivity in medical decision making. Determinant aspects of good guidelines, mainly, development methods, discussing, applicability factors such as costs, ethical and legal implications are approached in this article.

DESCRIPTORS: Guidelines. Clinical practice recommendations. Medical ethics.

\section{REFERÊNCIAS BIBLIOGRÁFICAS}

1 Evidence-Based Medicine Working Group - A new approach to teaching the practice of medicine. JAMA 1992; 268: 2420-5.

2 Heffner JE - Does evidence-based mediciine help the development of clinical pratice guidelines? Chest 1998; 113(3 suppl): 172-8S

3 Haycox A, Bagust A, Walley T-Clinical guidelines: the hidden costs. BMJ 1999; 318: 391-3.
4 Fletcher $\mathrm{RH}$ - Practice guidelines and the practice of medicine: is it the end of clinical judgment and expertise? Schweiz Med Wochemscher 1998; 128: 1883-8.

5 Berger JT \& Rosner F - The ethics of practice guidelines. Arch Intern Med 1996; 156: 2051-6.

6 Hyams AL, Brandenburg JA, Lipsitz SR et al. - Practice guidelines and inalpractice litigation: a two way street. Ann Intern Med 1995; 122(6): 450-455. 